\title{
Rodent brain slice model for the study of white matter injury
}

\author{
Akira Murata, MD, ${ }^{\mathrm{a}, \mathrm{b}}$ Kota Agematsu, MD, PhD, ${ }^{\mathrm{a}, \mathrm{b}}$ Ludmila Korotcova, MD, ${ }^{\mathrm{a}, \mathrm{b}}$ Vittorio Gallo, PhD, ${ }^{\mathrm{b}}$ \\ Richard A. Jonas, MD, ${ }^{\mathrm{a}, \mathrm{b}}$ and Nobuyuki Ishibashi, $\mathrm{MD}^{\mathrm{a}, \mathrm{b}}$
}

Objective: Cerebral white matter (WM) injury is common after cardiac surgery in neonates and young infants who have brain immaturity and genetic abnormalities. To understand better the mechanisms associated with WM injury, we tested the adequacy of a novel ex vivo brain slice model, with a particular focus on how the maturational stage modulates the injury.

Methods: To replicate conditions of cardiopulmonary bypass, we transferred living brain slices to a closed
chamber perfused by artificial cerebrospinal fluid under controlled temperature and oxygenation. Oxygen-
glucose deprivation (OGD) simulated circulatory arrest. The effects of maturation were investigated in 7- and
21-day-old mice (P7, P21) that are equivalent in maturation stage to the human fetus and young adult.

Results: There were no morphologic changes in axons after 60 minutes of OGD at $15^{\circ} \mathrm{C}$ in both P7 WM and P21 WM. Higher temperature and longer duration of OGD were associated with significantly greater WM axonal damage, suggesting that the model replicates the injury seen after hypothermic circulatory arrest. The axonal damage at P7 was significantly less than at P21, demonstrating that immature axons are more resistant than mature axons. Conversely, a significant increase in caspase $3^{+}$oligodendrocytes in P7 mice was identified relative to P21, indicating that oligodendrocytes in immature WM are more vulnerable than oligodendrocytes in mature WM.

Conclusions: Neuroprotective strategies for immature WM may need to focus on reducing oligodendrocyte injury. The brain slice model will be helpful in understanding the effects of cardiac surgery on the immature brain and the brain with genetic abnormalities. (J Thorac Cardiovasc Surg 2013;146:1526-33)

Supplemental material is available online.

Both prospective clinical trials and retrospective clinical studies have documented significant neurodevelopmental deficits in children with congenital heart disease (CHD). ${ }^{1-3}$ Recent studies using magnetic resonance imaging have identified evidence of white matter (WM) injury in neonates and young infants with CHD. ${ }^{4-6}$ Major clinical correlates of WM injury are gross and fine motor deficits, which are the most common neurologic deficits seen in children after cardiac surgery. ${ }^{7-9}$ In addition, recent advances in the field of neuroscience illuminate an

From the Children's National Heart Institute ${ }^{\mathrm{a}}$ and the Center for Neuroscience Research, ${ }^{\mathrm{b}}$ Children's National Medical Center, Washington, DC.

Funded by NIH R01HL060922 (to R.A.J.), R01HL104173 (to R.A.J.), R01NS045702 (to V.G.), P30HD040677 (to V.G.), and P01 NS0626860 (to V.G.).

R.A.J. and N.I. are joint senior authors.

Disclosures: Authors have nothing to disclose with regard to commercial support.

Received for publication Dec 25, 2012; revisions received Feb 13, 2013; accepted for publication Feb 28, 2013; available ahead of print March 29, 2013.

Address for reprints: Nobuyuki Ishibashi, MD, Children's National Medical Center, 111 Michigan Ave, NW, Washington, DC 20010-2970 (E-mail: nishibas@cnmc. org).

0022-5223/ $\$ 36.00$

Copyright (C) 2013 by The American Association for Thoracic Surgery

http://dx.doi.org/10.1016/j.jtcvs.2013.02.071 important role of WM structure in specific cognitive functions, including reading, verbal function, executive decision making, working memory, and learning complex skill. ${ }^{10}$ Interestingly, impairments of these functions are largely observed in school-aged children and adolescents who have had cardiac surgery for CHD. ${ }^{2,8,11}$ Therefore, understanding the cellular and molecular events that result in such WM injury is of crucial importance to develop targeted therapies and conditions that will minimize the risk of neurologic deficits in patients with CHD. ${ }^{12}$

Cellular and molecular processes underlying WM injury and its repair have been extensively explored in rodent animal models based on the tremendous power of transgenic and gene knockout technologies. ${ }^{13}$ This approach has assisted in establishing novel therapeutic strategies for WM disorders such as multiple sclerosis. ${ }^{14}$ We have recently introduced cutting-edge neuroscience techniques to study WM injury in the porcine model ${ }^{15}$; however, transgenic and gene knockout technologies are still extremely limited in large animals. Thus, for further investigation of WM injury and the development of novel treatment approaches, it is imperative to explore new animal models that replicate pathologic conditions to which the brain is exposed under CHD and subsequent cardiac surgery.

Clinical studies have reported a significant high incidence $(25 \%-55 \%)$ of newly developed WM injury after cardiac surgery. ${ }^{4-6}$ The major brain insults during surgery include 

Abbreviations and Acronyms
aCSF $=$ artificial cerebrospinal fluid
CHD = congenital heart disease
$\mathrm{CNP}=2^{\prime}, 3^{\prime}$-cyclic nucleotide
$3^{\prime}$ phosphodiesterase
$\mathrm{CPB}=$ cardiopulmonary bypass
DHCA $=$ deep hypothermic circulatory arrest
OGD = oxygen-glucose deprivation
PBS = phosphate-buffered saline
$\mathrm{WM}=$ white matter

cardiopulmonary bypass (CPB) and deep hypothermic circulatory arrest (DHCA). ${ }^{8,16}$ DHCA is a unique and specific pathologic condition in patients undergoing cardiac surgery, which causes ischemia-reperfusion/ reoxygenation under hypothermia. To reproduce conditions of CPB and DHCA, we developed a unique brain slice model in which living brain slices are transferred to a closed circulation system perfused by artificial cerebrospinal fluid (aCSF) under controlled temperature, $\mathrm{pH}$, and oxygenation. In the present study, we examined the adequacy of our brain slice model for the investigation of WM injury associated with DHCA. We assessed WM injury owing to hypothermic ischemia-reperfusion/reoxygenation using 2 transgenic mice strains with a focus on axons and oligodendrocytes, which are major cellular components in the WM. ${ }^{13,14}$ Inasmuch as recent clinical studies identified brain maturation as an important factor in WM injury after cardiac surgery, ${ }^{5}$ we studied how maturation stage modulates the damage in WM axons and oligodendrocytes.

\section{METHODS}

\section{Animals}

Two lines of transgenic mice were studied. In thy1-yellow fluorescent protein-16 (C57BL/6) mice, yellow fluorescent proteins are selectively expressed in neuronal bodies and axons. In $2^{\prime}, 3^{\prime}$-cyclic nucleotide $3^{\prime}$ phosphodiesterase (CNP) mice, human enhanced green fluorescent protein is overexpressed in the oligodendrocyte lineage under the CNP promoter.

Brain maturation in newborns with CHD is delayed approximately 1 month. ${ }^{17,18}$ Therefore, the effects of brain maturation on WM injury were investigated in both 7- and 21-day-old mice (P7, P21) that have WM maturation equivalent to the human fetus (35-36 weeks of gestation) and to a 10 -year-old child, respectively. ${ }^{19}$ We performed all experiments in compliance with the "Guide to the Care and Use of Laboratory Animals" published by the National Institutes of Health. The study was approved by the Animal Care and Use Committee of the Children's National Medical Center.

\section{Brain Slice Preparation}

The brains were collected from 2 transgenic mice strains at P7 and P21 and were dissected out into ice-cold $95 \%$ oxygenated modified aCSF slicing solution composed of the following (in millimoles per liter): $87 \mathrm{NaCl}$, $2.5 \mathrm{KCl}, 3 \mathrm{MgCl}_{2}, 0.5 \mathrm{CaCl}_{2}, 1.25 \mathrm{NaH}_{2} \mathrm{PO}_{4}, 25 \mathrm{NaHCO}_{3}, 25$ glucose, and 75 sucrose, $\mathrm{pH}$ 7.4. Coronal sections, $400 \mu \mathrm{m}$, were cut using a vibratome (Leica 1200 VT; Leica Microsystme Inc, Buffalo Grove, Ill). Slices were allowed to recover at $22^{\circ} \mathrm{C}$ in the slicing solution for 1 hour. Perfusate temperature was then gradually increased up to $36^{\circ} \mathrm{C}$ over 2 hours as is the practice for electrophysiologic studies using murine brain slices. ${ }^{20}$ Only slices containing corpus callosum, which is a major WM structure in the mouse brain, were included in the experiments (2-3 per animal). The slices from 3 littermates (6-9 per experiment) were transferred to a customized tissue chamber (CellMicro, Norfork, Va; Figure 1, A and B).

In the closed chamber system, brain slices were perfused $(2 \mathrm{~mL} / \mathrm{min})$ with aCSF composed of the following (in millimoles per liter): 126 $\mathrm{NaCl}, 3.5 \mathrm{KCl}, 1.3 \mathrm{MgCl}_{2}, 2 \mathrm{CaCl}_{2}, 1.2 \mathrm{NaH}_{2} \mathrm{PO}_{4}, 25 \mathrm{NaHCO}_{3}$, and 10 glucose, $\mathrm{pH} 7.4$; saturated with $95 \%$ oxygen $/ 5 \%$ carbon dioxide. A heating/ cooling device (CellMicro, Norfork, Va) was connected with the system to control the temperature of the aCSF. The bottom of the chamber itself also contained the temperature-control device (CellMicro). This bipolar temperature controller allows precise temperature control in slices being studied. Brain slice preparation and perfusion methods were modified from previous reports. 20,21

\section{Oxygen-Glucose Deprivation}

To replicate DHCA, we exposed slices to oxygen-glucose deprivation (OGD) (Figure 1, A). OGD was achieved by changing the perfusion solution from aCSF saturated with $95 \%$ oxygen $/ 5 \%$ carbon dioxide to glucosefree aCSF (supplemented with $10 \mathrm{mmol} / \mathrm{L}$ sucrose to maintain osmolarity) saturated with $95 \%$ nitrogen $/ 5 \%$ carbon dioxide. The slice was reperfused with glucose-containing aCSF saturated with $95 \%$ oxygen $/ 5 \%$ carbon dioxide after the period of OGD, providing ischemia-reperfusion/ reoxygenation to the brain slices. This technique has been widely used to investigate ischemia-induced brain injury in culture systems. ${ }^{21,22}$ In the present study, the OGD was performed under 3 hypothermic conditions using the temperature-controlled circulation system described above. The contents of oxygenated aCSF and aCSF under OGD are shown in Table E1.

\section{Perfusion Protocol}

Each experiment was assigned to 1 of 3 groups with different temperatures of 60 minutes of OGD $\left(15^{\circ} \mathrm{C}, 25^{\circ} \mathrm{C}\right.$, and $360 \mathrm{C}$ OGD). After the initial recovery period after the slice procedure, brain slices were cooled to a temperature of $15^{\circ} \mathrm{C}, 25^{\circ} \mathrm{C}$, or $36^{\circ} \mathrm{C}$ for 30 minutes according to the protocol. The OGD was then performed for 60 minutes under 3 temperature settings. After the OGD, slices were reperfused with glucose containing aCSF saturated with $95 \%$ oxygen $/ 5 \%$ carbon dioxide in 5 minutes, followed by a 30-minute rewarming period (Figure 1,C). This replicates DHCAinduced hypothermic ischemia-reperfusion/reoxygenation. Damage to WM axons and oligodendrocytes in brain slices was assessed at 0 to 20 hours after rewarming. Control slices, which were perfused at $36^{\circ} \mathrm{C}$ with aCSF saturated with $95 \%$ oxygen $/ 5 \%$ carbon dioxide for the same duration (no-OGD), were used for comparison with the OGD groups.

\section{Tissue Preparation and Immunohistochemistry}

At the conclusion of each experiment, slices were fixed in $4 \%$ paraformaldehyde in $0.1 \mathrm{~mol} / \mathrm{L}$ phosphate-buffered saline (PBS) for 1 hour at room temperature. Each slice was then cryoprotected for 48 hours in $30 \%$ sucrose in PBS. All slices were stored at $-80^{\circ} \mathrm{C}$ until further processing. For assessment of WM axonal and oligodendrocyte injury, 20- $\mu \mathrm{m}$ sections were developed from each $400-\mu \mathrm{m}$ brain slice using a cryotome. Sections from the outer $100 \mu \mathrm{m}$ of each slice were excluded from the assessment to avoid tissues that may have been damaged during slice preparation. For immunohistochemistry, 20- $\mu \mathrm{m}$ sections were incubated for 1 hour at room temperature with blocking solution ( $10 \%$ normal goat serum, $1 \%$ bovine serum albumin, and $0.3 \%$ Tween 20 in PBS, pH 7.4) and then incubated at $4{ }^{\circ} \mathrm{C}$ overnight with primary antibody and carrier solution $(1 \%$ normal goat serum, $1 \%$ bovine serum albumin, and $0.3 \%$ Tween 20 in PBS, pH 7.4). Sections were washed with PBS and then incubated for 1 hour at room temperature with secondary antibody and carrier solution. ${ }^{15,20}$ An antibody to cleaved caspase- 3 was used to identify apoptosis in the WM. ${ }^{15}$ 

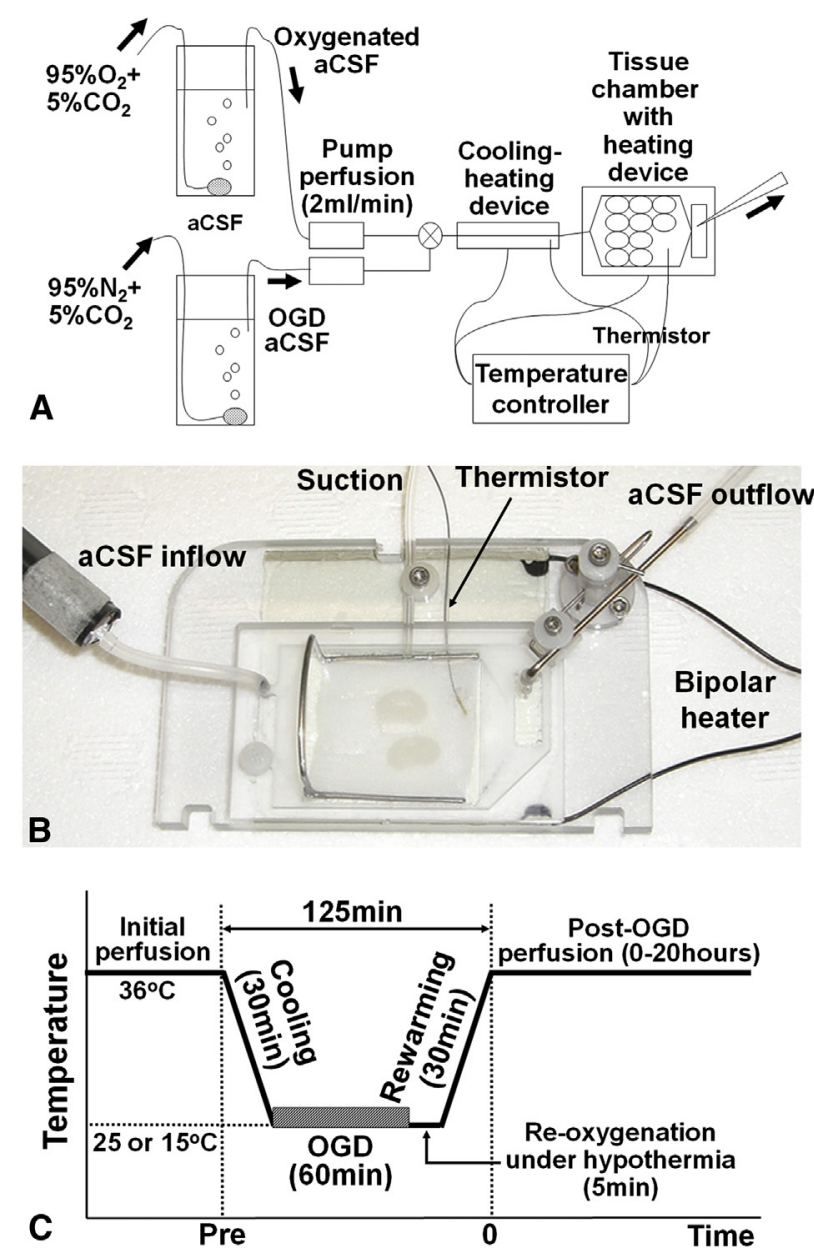

FIGURE 1. A, Schematic image of ex vivo brain slice culture system. B, Tissue chamber for rodent brain slices with controlled perfusion and temperature. C, Perfusion protocol of the oxygen-glucose deprivation $(O G D)$ under the hypothermia to replicate brain conditions under deep hypothermic circulatory arrest. $a C S F$, Artificial cerebrospinal fluid.

\section{Assessments of WM Cellular Damage}

A Zeiss LSM 510 confocal laser scanning microscopic system (Carl Zeiss Microimaging LLC, Thornwood, NY) was used for the analysis of fluorescence. Three different laser lines were used to image localization of fluorescein isothiocyanate (488-nm excitation; 522/35 emission filter), cyanine (Сy3; 560-nm excitation; 605/32 emission filter), and 4',6-diamidino-2-phenylindole (DAPI; 400-nm excitation). Data acquisition and processing were controlled by laser scanning microscopic software. Immunofluoresence was analyzed on confocal z-stacks. Confocal Assistant 4.02 and NIH Image J software (http://rsb.info.nih.gov) were used to merge images for analysis. Merged images were processed in Photoshop 7.0 (Adobe Systems; San Jose, Calif) with minimal manipulation of contrast as previously described. ${ }^{15,20}$

For the assessment of WM axonal damage, a total of 3 optical images $45 \times 45 \times 2 \mu \mathrm{m}(\mathrm{X}, \mathrm{Y}, \mathrm{Z}$ planes) were collected in the $\mathrm{z}$-axis from a single microscopic field in the midline of the corpus callosum of each section using a $100 \times$ oil immersion objective lens. The images including all tested groups were randomized and renamed by Ant Renamer 2.09.1. Randomized images were divided into a $5 \times 5$ grid (a total of 25 grids in a single image) and blindly scored by a single observer using the following scoring system: 0 , no damage; 1 , axon swelling and/or beading; and 2, axon fragmentation and/or loss of fluorescence, as previously described. ${ }^{23}$ The total score for a single image was 0 to $50(50=2 \times 25$ grids $)$. The numbers from 3 images were averaged and used as damage score of a single section. $\mathrm{WM}$ oligodendrocyte injury was assessed by counting $\mathrm{CNP}^{+}$caspase- $3^{+}$ cell numbers in $225 \times 225 \times 20 \mu \mathrm{m}(\mathrm{X}, \mathrm{Y}, \mathrm{Z}$ planes) for cell/volume quantifications. To determine cell density, we quantified the antibody positive cells in 3 microscopic fields from the corpus callosum of each brain slice as previously described. ${ }^{15,20}$

\section{Statistical Analysis}

One-way analysis of variance with Bonferroni post hoc comparisons was used to detect differences in the axonal damage score and caspase $3^{+} \mathrm{CNP}^{+}$cell number in the experimental groups. Two-group comparisons in the OGD conditions and ages were performed by unpaired Student $t$ tests. Two-way analysis of variance with Bonferroni comparisons was used to evaluate changes in axonal damage score over time between OGD groups. The Spearman rank correlation coefficient (rho) was used as a measure of the association between cellular damage and OGD temperature and OGD duration. All data are expressed as mean \pm standard error of the mean. Reported $P$ values are 2-tailed. Analysis of the data was performed using SPSS version 19.0 (SPSS Inc/IBM, Chicago, Ill).

\section{RESULTS \\ Ex Vivo Brain Slice Model Replicates DHCA-Induced WM Injury in a Rodent Animal}

To test the adequacy of brain slice model for investigation of DHCA-induced WM injury, we first assessed WM axonal injury using thy 1-YFP mice. Morphologic changes in the axons including beading and fragmentation are sensitive markers of WM axonal injury. ${ }^{23}$ In our model, WM axons in P21 control slices maintained normal morphologic structures until the end of the experiment (Figure 2, $A-D$ ). There was no significant increase in damage score in the control slices over time (Figure 2, $Q$ ), indicating that the aCSF circulation system is able to maintain the morphologic structure of WM axons. On the other hand, $25^{\circ} \mathrm{C}$ and $36^{\circ} \mathrm{C}$ OGD induced significant alterations including diffuse swelling, beading, and fragmentation (Figure 2, $I-P$ ). The morphologic alterations and damage score after $25^{\circ} \mathrm{C}$ and $36^{\circ} \mathrm{C}$ OGD significantly increased over time (Figure 2, $Q$ ). Damage observed with 60 minutes' OGD, however, was not observed with OGD under deep hypothermia at $15^{\circ} \mathrm{C}$ compared with control (Figure 2, E-H, $Q$ ). The protective effects of hypothermia at $15^{\circ} \mathrm{C}$ have been noted previously in a porcine model of DHCA. ${ }^{16}$ The OGD-induced WM injury in our ex vivo model demonstrated a significant positive correlation with temperature during OGD (Figure 2, $R$ ). Furthermore, longer OGD duration was associated with significantly higher WM damage score at $25^{\circ} \mathrm{C}$ (Figure 2, $S$ ). These observations are consistent with the results of DHCA-induced brain injury in a large animal model. ${ }^{16}$

\section{Axons in Immature WM Are More Resistant to Insults Than Mature WM}

Newborns with CHD born at term have a delay in brain development of approximately 1 month. ${ }^{17,18}$ Furthermore, brain immaturity is associated with WM injury after 

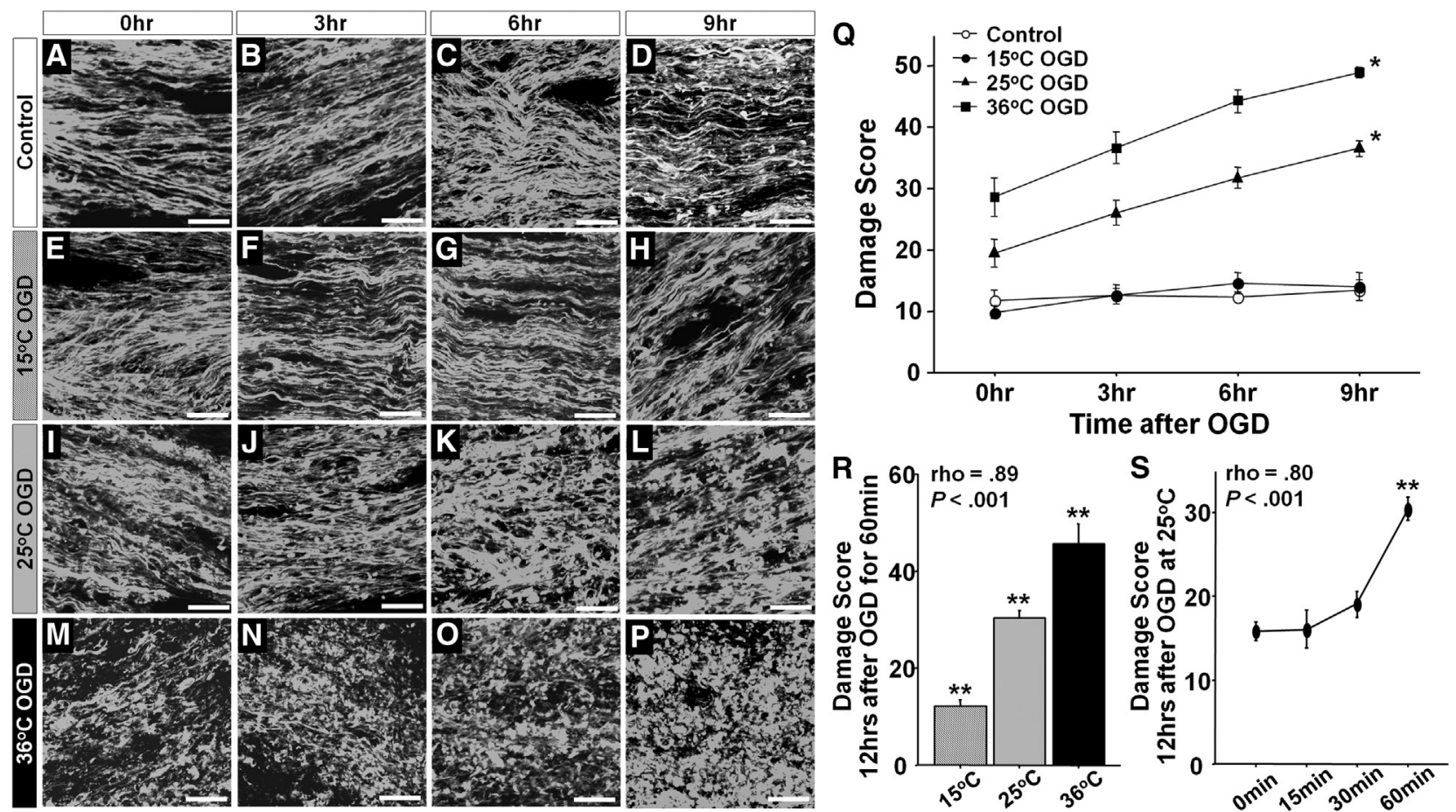

FIGURE 2. A-P, Morphologic changes of WM axons at $0,3,6$, and 9 hours after experiments in control (A-D), $15^{\circ} \mathrm{C}$ OGD (E-H), $25^{\circ} \mathrm{C}$ OGD (I-L), and $36^{\circ} \mathrm{C} \mathrm{OGD} \mathrm{(M-P).} \mathrm{Q,} \mathrm{Damage} \mathrm{scores} \mathrm{at} 0,3,6$, and 9 hours after experiments in control, $15^{\circ} \mathrm{C}, 25^{\circ} \mathrm{C}$, and $36^{\circ} \mathrm{C} \mathrm{OGD}$. The scores differ according to the time after the OGD $(\mathrm{F}=20.0 ; P<.001)$ and 4 OGD groups $(\mathrm{F}=155.2 ; P<.001)$, independently $(\mathrm{F}=4.0 ; P<.001 ; \mathrm{n}=6-13$ each). There are no significant differences of the score between control and $15^{\circ} \mathrm{C} \mathrm{OGD}(P=.84)$. R, The damage score at 12 hours after 60 minutes of OGD at 3 temperature groups. There are significant differences among 3 temperature $\left(\mathrm{F}=60.7 ; P<.001 ; \mathrm{n}=12,16\right.$, and 6 in $15^{\circ} \mathrm{C}, 25^{\circ} \mathrm{C}$, and $36^{\circ} \mathrm{C}$ OGD, respectively). The scores are significantly correlated with temperature during OGD. S, The damage score at 12 hours after $25^{\circ} \mathrm{C}$ OGD at 4 duration groups. There are significant differences between 4 OGD durations $(\mathrm{F}=26.3 ; P<.001 ; \mathrm{n}=12,6,6$, and 17 in $0,15,30$, and 60 minutes, respectively). The scores are significantly correlated with the OGD duration. $* P<.001$ versus other 3 groups by 2 -way analysis of variance with Bonferroni adjusted comparisons. ** $P<.001$ versus other groups by 1 way analysis of variance with Bonferroni comparisons. Scale bar $=10 \mu \mathrm{m}$. Data are shown as mean \pm standard error of the mean. WM, White matter; $O G D$, oxygen-glucose deprivation.

surgery. ${ }^{5}$ On the basis of oligodendrocyte lineage progression and myelination, it has been confirmed that WM maturation in $\mathrm{P} 7$ mice is equivalent to the human fetus at 35 to 36 weeks' gestation. ${ }^{19}$ We therefore investigated the effects of WM maturation on DHCA-induced axonal injury using P7 and P21 murine brain slices.

Consistent with the findings in P21 WM, significant degeneration of $\mathrm{WM}$ axons and an increase in damage score were observed at 12 hours after $25^{\circ} \mathrm{C}$ and $36^{\circ} \mathrm{C}$ OGD compared with control, but not after $15^{\circ} \mathrm{C}$ OGD (Figure $3, A-F$, $I$, and $L$ and Table 1). Longer OGD duration at $25^{\circ} \mathrm{C}$ in $\mathrm{P} 7$ slices was significantly correlated with a higher damage score as observed in P21 slices (Figure 3, M). In P7 WM, morphologic degeneration owing to OGD-induced insult was significantly less compared with in $\mathrm{P} 21$ slices for all temperature groups (Figure 3, $A-L$ ). In P21 WM, significant axonal beading and fragmentation and increase in the damage score were identified immediately after $36^{\circ} \mathrm{C}$ OGD (Figure 3, $G, H$, and $N$ ); however, these observations were not seen in P7 WM (Figure 3, $J, K$, and $O$ ). At 12 hours after $36^{\circ} \mathrm{C}$ OGD, there was extensive fragmentation in P21 WM
(Figure 3,L). On the other hand, significant alterations were not identified in $\mathrm{P} 7 \mathrm{WM}$ at 12 hours after $36^{\circ} \mathrm{C}$ OGD (Figure $3, I$ ). In this age group, beading and swelling were observed; however, the morphologic structure of axons was still maintained (Figure 3,I). When the axonal damage score and the change from control were compared between P7 and P21, a significant decrease in both parameters at 12 hours after $36^{\circ} \mathrm{C}$ OGD was identified in P7 WM compared with $\mathrm{P} 21$ (Figure 3, $P$ and $Q$ ). Overall, these results indicate that the axons in immature WM are more resistant to ischemia-reperfusion/reoxygenation injury compared with mature WM.

\section{Oligodendrocytes in Immature WM Are More Vulnerable Than Oligodendrocytes in the Mature Brain}

We next investigated WM oligodendrocyte injury after OGD-induced insults. Consistent with findings in WM axonal injury, there were significant positive correlations between $\mathrm{CNP}^{+}$caspase $3^{+}$cell number and temperatures during OGD in both P7 WM and P21 WM (Figure 4, $A-H$ ). 

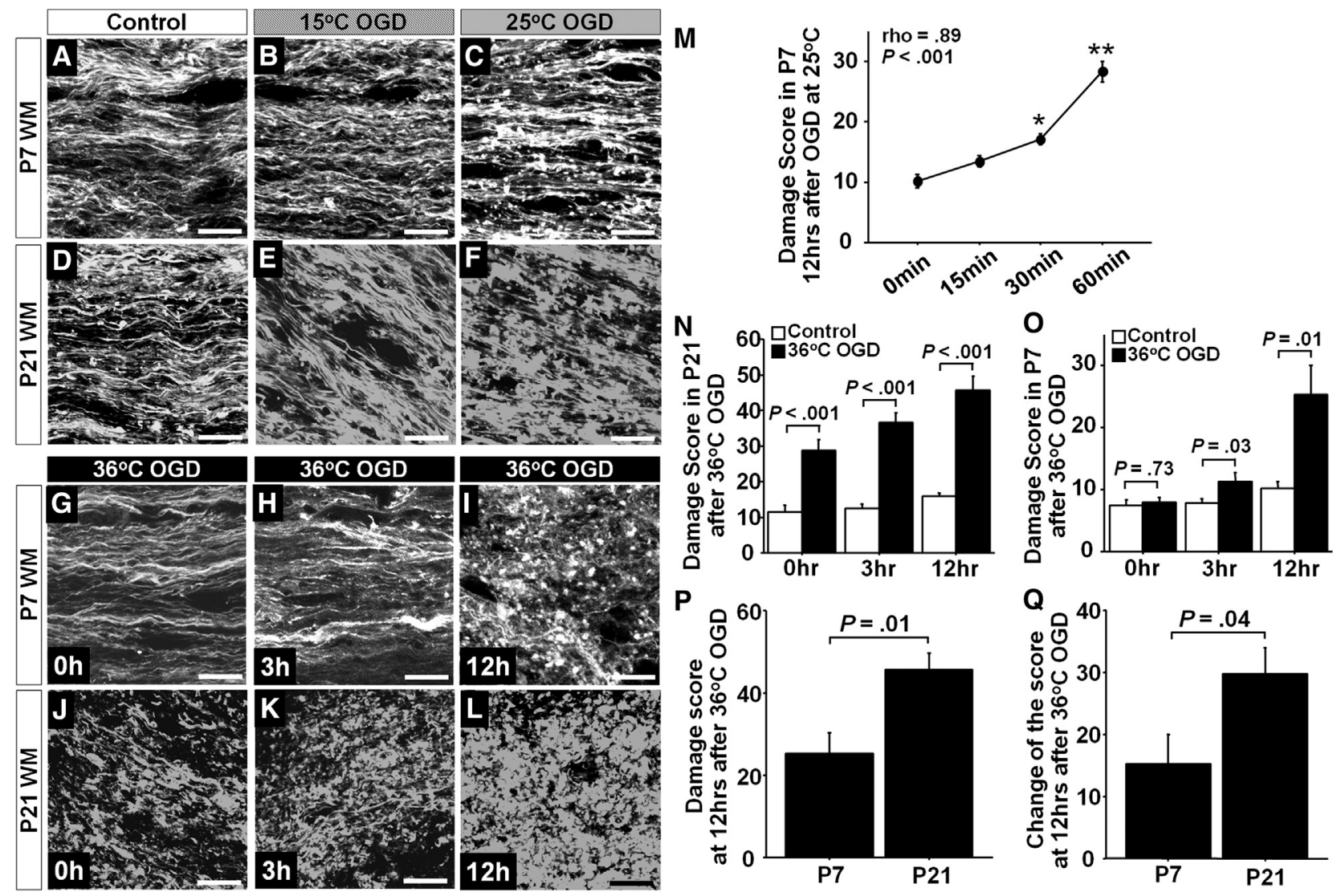

FIGURE 3. A-F, Morphologic changes of WM axons between $\mathrm{P} 7$ (A-C) and $\mathrm{P} 21$ (D-F) at 12 hours after experiments in control (A and D), $15^{\circ} \mathrm{C} \mathrm{OGD} \mathrm{(B}$ and $\mathrm{E}$ ), and $25^{\circ} \mathrm{C}$ OGD (C and F). G-L, Morphological changes of WM axons at 0, 3, and 12 hours after $36^{\circ} \mathrm{C}$ OGD in P7 WM (G-I) and P21 WM (J-L). M, Axonal damage score in $\mathrm{P} 7 \mathrm{WM}$ at 12 hours after $25^{\circ} \mathrm{C}$ OGD among 4 OGD durations. There were significant differences among the 4 groups $(\mathrm{F}=36.6$; $P<.001 ; \mathrm{n}=6,9,9$, and 11 at $0,15,30$, and 60 minutes, respectively). The scores are significantly correlated with the OGD duration. N, Axonal damage score in $\mathrm{P} 21 \mathrm{WM}$ at 0,3 , and 12 hours after $36^{\circ} \mathrm{C} \mathrm{OGD}$ and control experiment $\left(\mathrm{n}=6-12\right.$ each in control; $\mathrm{n}=6-9$ each in $36^{\circ} \mathrm{C}$ OGD). O, Axonal damage score in $\mathrm{P} 7 \mathrm{WM}$ at 0,3 , and 12 hours after $36^{\circ} \mathrm{C}$ OGD and control experiment ( $\mathrm{n}=6-12$ each in control; $\mathrm{n}=6$ each in $36^{\circ} \mathrm{C}$ OGD). $\mathrm{P}$, The axonal damage score after $36^{\circ} \mathrm{C}$ OGD between P7 and P21 WM $\left(\mathrm{n}=6\right.$ each). Q, The change in axonal damage score after $36^{\circ} \mathrm{C}$ OGD from control between P7 and P21 WM $(\mathrm{n}=6$ each). $* P<.01$ versus 0 minutes; $* * P<.0001$ versus 0,15 , and 30 minutes; analysis of variance with Bonferroni adjusted comparisons. Scale bar $=10$ $\mu \mathrm{m}$, Data are shown as mean \pm standard error of the mean. $W M$, White matter; $O G D$, oxygen-glucose deprivation.

$\mathrm{CNP}^{+}$caspase $3^{+}$cell numbers after $36^{\circ} \mathrm{C}$ OGD were significantly greater compared with other groups at both $\mathrm{P} 7$ and P21, as was observed with WM axonal injury (Figure 4, $G$ and $H$ ). There was a significant difference in the number of $\mathrm{CNP}^{+}$caspase $3^{+}$cells between $15^{\circ} \mathrm{C}$ and $25^{\circ} \mathrm{C}$ OGD in P21 WM (Figure 4, $H$ ); however, this was not seen in P7 slices (Figure 4, G). This indicates that oligodendrocyte response to hypothermia varies according to WM maturational stage.

To identify how maturational stages modulate oligodendrocyte injury, we compared $\mathrm{CNP}^{+}$caspase $3^{+}$cell numbers after $36^{\circ} \mathrm{C}$ OGD between P7 WM and P21 WM. In WM

TABLE 1. Comparison of WM axonal and oligodendrocyte injury

\begin{tabular}{|c|c|c|c|c|c|c|c|c|c|c|c|c|}
\hline & Control & $15^{\circ} \mathrm{C}$ OGD & $25^{\circ} \mathrm{C}$ OGD & $36^{\circ} \mathrm{C}$ OGD & F value & $P$ value & 1 & 2 & 3 & 4 & 5 & 6 \\
\hline \multicolumn{13}{|c|}{ Axonal damage score } \\
\hline P7 & $10.2 \pm 2.8$ & $9.1 \pm 2.4$ & $28.4 \pm 5.8$ & $25.3 \pm 11.8$ & 16.8 & $<.001$ & .785 & $<.001$ & $<.001$ & $<.001$ & $<.001$ & .376 \\
\hline $\mathrm{P} 21$ & $15.9 \pm 3.6$ & $12.3 \pm 4.3$ & $30.3 \pm 5.9$ & $45.7 \pm 10.1$ & 59.6 & $<.001$ & .134 & $<.001$ & $<.001$ & $<.001$ & $<.001$ & $<.001$ \\
\hline \multicolumn{13}{|c|}{ Caspase $^{+} \mathrm{CNP}^{+}$cell number per $10^{6} \mu \mathrm{m}$} \\
\hline P7 & $8.6 \pm 1.4$ & $6.8 \pm 4.0$ & $8.7 \pm 4.3$ & $21.0 \pm 6.7$ & 26.5 & $<.001$ & .437 & .977 & $<.001$ & .330 & $<.001$ & $<.001$ \\
\hline P21 & $7.3 \pm 2.9$ & $5.4 \pm 1.7$ & $9.7 \pm 3.5$ & $14.5 \pm 4.3$ & 18.3 & $<.001$ & .176 & .100 & $<.001$ & $<.01$ & $<.001$ & $<.001$ \\
\hline
\end{tabular}

$W M$, White matter; $O G D$, oxygen-glucose deprivation; $P 7$, postnatal day $7 ; P 21$, postnatal day $21 ; 1$, control versus $15^{\circ} \mathrm{C}$ OGD; 2 , control versus $25^{\circ} \mathrm{C}$ OGD; 3 , control versus $36^{\circ} \mathrm{C}$ OGD; $4,15^{\circ} \mathrm{C}$ versus $25^{\circ} \mathrm{C} \mathrm{OGD} ; 5,15^{\circ} \mathrm{C}$ versus $36^{\circ} \mathrm{C}$ OGD; $6,25^{\circ} \mathrm{C}$ versus $36^{\circ} \mathrm{C}$ OGD by analysis of variance with Bonferroni comparisons. 

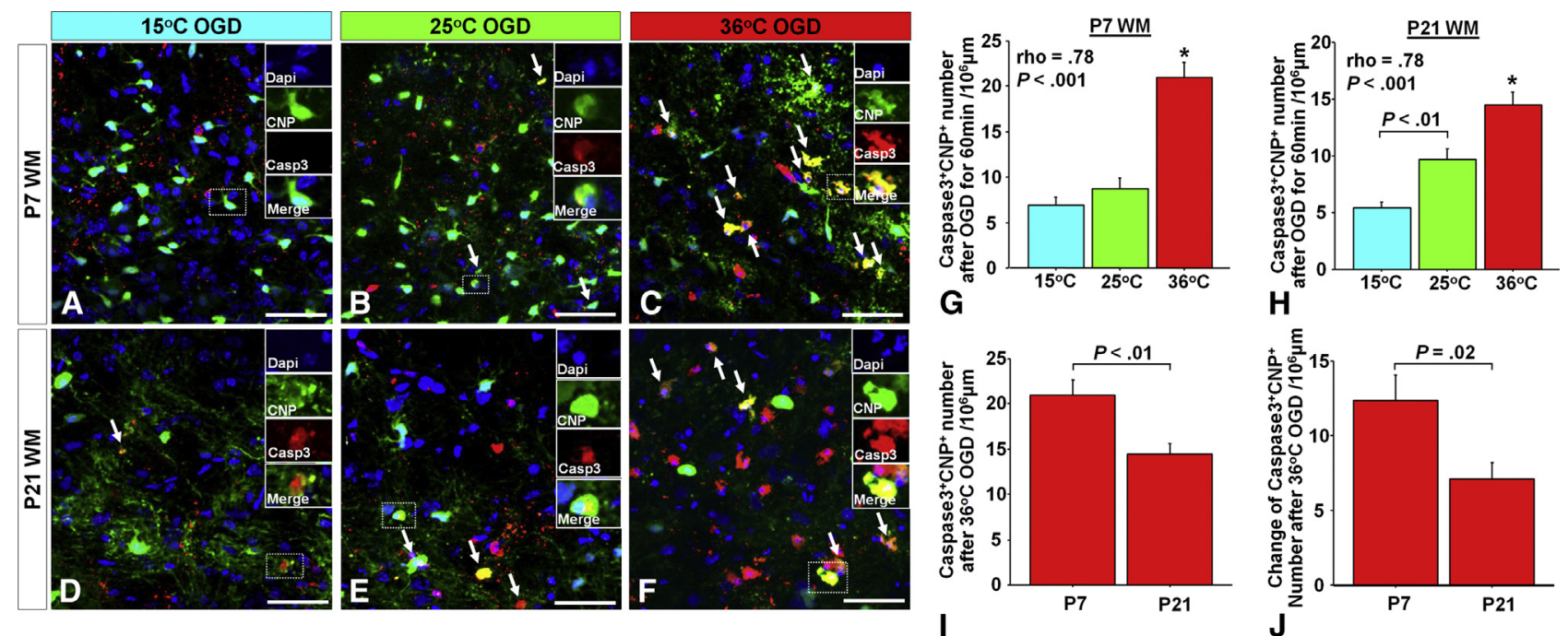

FIGURE 4. A-F, Images of caspase $3^{+}$oligodedrocytes in $\mathrm{P} 7$ and $\mathrm{P} 21 \mathrm{WM}$ between three OGD groups. $\mathrm{G}$ and $\mathrm{H}$, Caspase $3^{+} \mathrm{CNP}^{+}$cell number in $\mathrm{P} 7$ and $\mathrm{P} 21 \mathrm{WM}$ after 60 minutes of OGD among 3 temperature groups. There are significant differences among 3 groups in both $\mathrm{P} 7(\mathrm{~F}=33.4 ; P<.001 ; \mathrm{n}=15,13$, and 16 in $15^{\circ} \mathrm{C}, 25^{\circ} \mathrm{C}$, and $36^{\circ} \mathrm{C} \mathrm{OGD}$, respectively) and $\mathrm{P} 21\left(\mathrm{~F}=23.5 ; P<.001 ; \mathrm{n}=12,15\right.$, and 15 in $15^{\circ} \mathrm{C}, 25^{\circ} \mathrm{C}$, and $36^{\circ} \mathrm{C}$ OGD, respectively). The numbers are significantly correlated with temperature during OGD. I, Caspase $3^{+} \mathrm{CNP}^{+}$cell number after $36^{\circ} \mathrm{C}$ OGD for 60 minutes between P7 and P21 $\mathrm{WM}\left(\mathrm{n}=6\right.$ each). $\mathrm{J}$, Changes of caspase $3^{+} \mathrm{CNP}^{+}$cell number after $35^{\circ} \mathrm{C}$ OGD from control between $\mathrm{P} 7$ and $\mathrm{P} 21 \mathrm{WM}(\mathrm{n}=6$ each $) . P<.001$ versus other 2 groups by analysis of variance with Bonferroni comparisons. Arrows indicate caspase $3^{+} \mathrm{CNP}^{+}$cells. Scale bar $=50 \mu \mathrm{m}$, Data are shown as mean \pm standard error of the mean. $W M$, White matter; $O G D$, oxygen-glucose deprivation.

axons, there was a significant decrease in the damage in P7 WM compared with in P21 WM (Figure 3, $P$ and $Q$ ). Conversely a significant increase in the $\mathrm{CNP}^{+}$caspase $3^{+}$cell number as well as changes of the number from control were demonstrated in P7 compared with in P21 (Figure 4, $I$ and $J$ ), indicating that oligodendrocytes in the immature WM are more vulnerable than oligodendrocytes in the mature brain. These results suggest that oligodendrocytes should be the target cell population to develop novel protective strategies for immature WM.

\section{DISCUSSION}

This study is the first to describe an ex vivo mouse model replicating DHCA under specific brain conditions including ischemia-reperfusion/reoxygenation under hypothermia. We demonstrated temperature-dependent WM injury similar to observations in large animal models of DHCA. Using 2 transgenic mouse lines, our analysis has identified a likely cellular target, namely the oligodendrocyte, to reduce risks of WM injury in immature WM.

WM injury in neonates and infants with CHD has been the focus of several recent reports. ${ }^{4-6,12}$ Recent clinical studies suggest that brain maturation in CHD newborns is delayed approximately 1 month $^{17,18}$ and that immaturity increases susceptibility to WM injury after cardiac surgery. ${ }^{5}$ In the present study, the effects of WM maturation stage on injury was studied using P7 and P21 mice WM, which are equivalent in the human to 35 to 36 weeks' gestation and the 10-year-old child, respectively. ${ }^{19}$
Interestingly our results demonstrate that axons in immature WM are more resistant to ischemia-reperfusion/ reoxygenation injury compared with mature $\mathrm{WM}$; in contrast, oligodendrocytes are more susceptible in immature WM. Our previous study using a juvenile porcine model identified both WM oligodendrocyte injury and axonal damage after CPB-induced ischemia-reperfusion/reoxygenation injury. ${ }^{15}$ There has been controversy whether neuroaxonal elements or oligodendrocyte injury are a primary cause of hypomyelination in WM injury. ${ }^{24}$ However, we were not able to determine the primary cause of CPB-induced hypomyelination at 4 weeks after surgery in our porcine CPB model. ${ }^{15}$ Our results in this study suggest that the primary cellular target to prevent CPB-induced WM injury in immature brain is oligodendrocyte lineage cells.

Oligodendrocyte lineages are the cells responsible for myelin synthesis and for axonal myelination, which increases conduction velocity up to 300 times, allowing fast and efficient communication between neurons and within neural circuits. ${ }^{10,13}$ Cellular alterations in the oligodendrocyte development include oligodendrocyte death, delayed oligodendrocyte differentiation, abnormal myelination, and hypomyelination. ${ }^{13,14}$ Clinical findings of molecular imaging, such as higher average diffusivity and lower WM fractional anisotropy in CHD newborns with delayed brain maturation, are associated with alterations in the oligodendrocyte development and myelination. ${ }^{4,10}$ Within different oligodendrocyte developmental stages, we already showed selective vulnerability of pre-oligodendrocytes 
to CPB-induced brain insults in porcine CPB model. ${ }^{15}$ Thus, our understanding of cellular/molecular alterations of pre-oligodendrocytes resulted from many pathologic conditions including ischemia-reperfusion, reoxygenation, hypothermia, inflammation, and anesthesia will contribute to the development of optimal management strategies and adjunctive pharmacologic approaches to reduce risks of WM injury in neonates and young infants with CHD.

There is accumulating evidence that CHD patients requiring surgical correction using $\mathrm{CPB}$ and DHCA early in life are at significant risk of neurologic deficits. ${ }^{2,5,7}$ Causes of insults to the developing brain triggered by $\mathrm{CPB}$ and DHCA include (1) significant risk of ischemiareperfusion injury resulting from air or particulate emboli, blood steal by aortopulmonary collateral vessels, and possible cerebral vessel abnormalities; (2) reoxygenation and hypothermia caused by DHCA or selective cerebral perfusion; and (3) systemic inflammatory response owing to blood exposure to nonendothelial surfaces in the CPB system. ${ }^{8,16}$ Furthermore, it has been demonstrated that brain injury after cardiac surgery is affected by genetic abnormalities $^{25,26}$ and preoperative brain immaturity ${ }^{5}$ secondary to hypoxia in utero. ${ }^{12,27}$ Thus it is possible that CPB and DHCA management strategies that are optimal for the protection in the normally developing brain are damaging to the brain that has developed in the presence of genetic abnormalities, syndromes, and immaturities. Therefore, for further and more complete refinement of cardiac surgery management, it will be necessary to investigate the effects of CPB and DHCA-induced insults on the brain with genetic abnormalities or immaturity.

The results of the present study demonstrating temperature-dependent injury are similar to observations using a porcine DHCA model, ${ }^{16}$ suggesting that this model is suitable for investigation of brain injury resulting from hypothermic circulatory arrest. Inasmuch as the ex vivo model uses rodents, it allows us to investigate the effect of DHCA on genetically manipulated brains and the immature brain. In the present study, 2 lines of transgenic mice at 2 different ages, either with or without brain immaturity, were used to investigate the effects of ischemiareperfusion/reoxygenation under different degrees of hypothermia. Within mechanisms of brain insults owing to CPB and DHCA, we did not include effects of the inflammatory response in the present study. However, inasmuch as cellular and molecular mechanisms of the brain inflammation have been investigated using a brain slice model. ${ }^{28} \mathrm{We}$ are anticipating that this model will be suitable for study of other mechanisms of brain injury related to cardiac surgery, including inflammation. Our future study will investigate effects of inflammatory factors during and after OGD-induced ischemia-reperfusion and reoxygenation in this model. Rodent brain slice models have been used widely for the development of pharmacologic therapies for neurologic disorders. ${ }^{29}$ Thus the model will also be useful for the study of optimal anesthetic strategy in cardiac surgery, the need for which has been suggested by population-based developmental testing in children after noncardiac surgery, which has raised concern over optimal anesthetic strategies in children. ${ }^{27}$

The brain slice model is certainly different from CPB and DHCA and is not suitable to investigate effects of hemodynamic changes in the cerebral circulation or blood-brain barrier disruption. ${ }^{30,31}$ Compared with brain conditions in neonates and young infants during CPB and DHCA, this ex vivo system we described differs in extracellular volume or diffusion characteristics and constituents of extracellular brain volume. For investigation of these effects, an in vivo model using a large animal such as the piglet is beneficial for laboratory study. ${ }^{15}$ Mechanisms underlying brain insults during CPB and DHCA are complex and multietiologic. ${ }^{8,16}$ In contrast to an in vivo model, the brain slice model allows us to study each factor individually and helps in our understanding of complex and multifactorial events on the developing brain, such as our investigation of ischemia-reperfusion/reoxygenation under hypothermia in this study. Future studies using both rodent brain slice model and an in vivo large animal model will provide novel insights of cellular and molecular mechanisms underlying neurologic deficits after CPB and DHCA and allow for the design of targeted therapies and conditions that will minimize the risk of neurodevelopmental deficits in CHD patients.

In conclusion, efforts to improve WM protection in individuals with an immature brain undergoing cardiac surgery should focus on reducing oligodendrocyte injury. Future studies using our ex vivo slice model will provide novel insights of the possible additive effects of genetic abnormalities and brain immaturity on CPB and DHCA-induced brain insults and allow for the design of targeted therapies to improve neurodevelopmental outcomes in patients undergoing cardiac surgery.

We thank Dr David Zurakowski for assistance with statistical analysis. We are thankful to Dr Jean-Marie Mangin for assistance in the preparation of the brain slice culture and to Joseph Scafidi for critical discussions on model development.

\section{References}

1. Kaltman JR, Andropoulos DB, Checchia PA, Gaynor JW, Hoffman TM, Laussen PC, et al. Report of the Pediatric Heart Network and National Heart, Lung, and Blood Institute Working Group on the perioperative management of congenital heart disease. Circulation. 2010;121:2766-72.

2. Bellinger DC, Wypij D, Rivkin MJ, DeMaso DR, Robertson RL Jr, DunbarMasterson C, et al. Adolescents with d-transposition of the great arteries corrected with the arterial switch procedure: neuropsychological assessment and structural brain imaging. Circulation. 2011;124:1361-9.

3. Marino BS, Lipkin PH, Newburger JW, Peacock G, Gerdes M, Gaynor JW, et al. Neurodevelopmental outcomes in children with congenital heart disease: evaluation and management-a scientific statement from the American Heart Association. Circulation. 2012;126:1143-72. 
4. Miller SP, McQuillen PS, Hamrick S, Xu D, Glidden DV, Charlton N, et al. Abnormal brain development in newborns with congenital heart disease. $N$ Engl $J$ Med. 2007;357:1928-38.

5. Andropoulos DB, Hunter JV, Nelson DP, Stayer SA, Stark AR, McKenzie ED, et al. Brain immaturity is associated with brain injury before and after neonatal cardiac surgery with high-flow bypass and cerebral oxygenation monitoring. J Thorac Cardiovasc Surg. 2010;139:543-56.

6. Galli KK, Zimmerman RA, Jarvik GP, Wernovsky G, Kuypers MK, Clancy RR, et al. Periventricular leukomalacia is common after neonatal cardiac surgery. J Thorac Cardiovasc Surg. 2004;127:692-704.

7. Bellinger DC, Jonas RA, Rappaport LA, Wypij D, Wernovsky G, Kuban KC, et al. Developmental and neurologic status of children after heart surgery with hypothermic circulatory arrest or low-flow cardiopulmonary bypass. $N \mathrm{Engl}$ J Med. 1995;332:549-55.

8. Wernovsky G. Current insights regarding neurological and developmental abnormalities in children and young adults with complex congenital cardiac disease. Cardiol Young. 2006;1(16 Suppl):92-104

9. Jonas RA, Wypij D, Roth SJ, Bellinger DC, Visconti KJ, du Plessis AJ, et al. The influence of hemodilution on outcome after hypothermic cardiopulmonary bypass: rResults of a randomized trial in infants. J Thorac Cardiovasc Surg. 2003; $126: 1765-74$

10. Fields RD. White matter in learning, cognition and psychiatric disorders. Trends Neurosci. 2008:31:361-70

11. Bellinger DC, Wypij D, duPlessis AJ, Rappaport LA, Jonas RA, Wernovsky G, et al. Neurodevelopmental status at eight years in children with dextrotransposition of the great arteries: the Boston Circulatory Arrest trial. J Thorac Cardiovasc Surg. 2003;126:1385-96

12. McQuillen PS, Miller SP. Congenital heart disease and brain development. Ann N Y Acad Sci. 2010;1184:68-86.

13. Emery B. Regulation of oligodendrocyte differentiation and myelination. Science. 2010;330:779-82.

14. Franklin RJ, Ffrench-Constant C. Remyelination in the CNS: from biology to therapy. Nat Rev Neurosci. 2008;9:839-55.

15. Ishibashi N, Scafidi J, Murata A, Korotcova L, Zurakowski D, Gallo V, et al. White matter protection in congenital heart surgery. Circulation. 2012;125: 859-71.

16. Jonas RA. Hypothermia, reduced flow and circulatory arrest. London: A Hodder Arnold Publication; 2004.

17. Licht DJ, Shera DM, Clancy RR, Wernovsky G, Montenegro LM, Nicolson SC, et al. Brain maturation is delayed in infants with complex congenital heart defects. J Thorac Cardiovasc Surg. 2009;137:529-36; discussion 536-7.
18. Limperopoulos C, Tworetzky W, McElhinney DB, Newburger JW, Brown DW, Robertson RL Jr, et al. Brain volume and metabolism in fetuses with congenital heart disease: evaluation with quantitative magnetic resonance imaging and spectroscopy. Circulation. 2010;121:26-33.

19. Craig A, Ling Luo N, Beardsley DJ, Wingate-Pearse N, Walker DW, Hohimer AR, et al. Quantitative analysis of perinatal rodent oligodendrocyte lineage progression and its correlation with human. Exp Neurol. 2003;181:231-40.

20. Mangin JM, Li P, Scafidi J, Gallo V. Experience-dependent regulation of NG2 progenitors in the developing barrel cortex. Nat Neurosci. 2012;15:1192-4.

21. Tekkok SB, Goldberg MP. Ampa/kainate receptor activation mediates hypoxic oligodendrocyte death and axonal injury in cerebral white matter. $J$ Neurosci. 2001;21:4237-48.

22. Deng W, Rosenberg PA, Volpe JJ, Jensen FE. Calcium-permeable ampa/kainate receptors mediate toxicity and preconditioning by oxygen-glucose deprivation in oligodendrocyte precursors. Proc Natl Acad Sci U S A. 2003;100:6801-6.

23. McCarran WJ, Goldberg MP. White matter axon vulnerability to ampa/kainate receptor-mediated ischemic injury is developmentally regulated. $J$ Neurosci 2007;27:4220-9

24. Volpe JJ. Brain injury in premature infants: a complex amalgam of destructive and developmental disturbances. Lancet Neurol. 2009;8:110-24.

25. Gaynor JW, Nord AS, Wernovsky G, Bernbaum J, Solot CB, Burnham N, et al Apolipoprotein e genotype modifies the risk of behavior problems after infant cardiac surgery. Pediatrics. 2009;124:241-50.

26. Burnham N, Ittenbach RF, Stallings VA, Gerdes M, Zackai E, Bernbaum J, et al Genetic factors are important determinants of impaired growth after infan cardiac surgery. J Thorac Cardiovasc Surg. 2010;140:144-9.

27. Tabbutt S, Gaynor JW, Newburger JW. Neurodevelopmental outcomes after congenital heart surgery and strategies for improvement. Curr Opin Cardiol. 2012; 27:82-91.

28. Bernardino L, Balosso S, Ravizza T, Marchi N, Ku G, Randle JC, et al. Inflammatory events in hippocampal slice cultures prime neuronal susceptibility to excitotoxic injury: a crucial role of $\mathrm{P} 2 \mathrm{X} 7$ receptor-mediated IL-1beta release J Neurochem. 2008;106:271-80.

29. Pena F. Organotypic cultures as tool to test long-term effects of chemicals on the nervous system. Curr Med Chem. 2010;17:987-1001.

30. Anttila V, Hagino I, Zurakowski D, Lidov HG, Jonas RA. Higher bypass temperature correlates with increased white cell activation in the cerebral microcirculation. J Thorac Cardiovasc Surg. 2004;127:1781-8.

31. Ishibashi N, Iwata Y, Okamura T, Zurakowski D, Lidov HG, Jonas RA. Differential neuronal vulnerability varies according to specific cardiopulmonary bypass insult in a porcine survival model. J Thorac Cardiovasc Surg. 2010;140: 1408-15.e1403. 
TABLE E1. Contents of aCSF

aCSF in baseline perfusion aCSF during OGD

\begin{tabular}{lcc}
\hline $\mathrm{pH}$ & $7.36 \pm 0.0$ & $7.31 \pm 0.0$ \\
$\mathrm{PCO}_{2}(\mathrm{~mm} \mathrm{Hg})$ & $46.2 \pm 0.9$ & $48.3 \pm 1.2$ \\
$\mathrm{Po}_{2}(\mathrm{~mm} \mathrm{Hg})$ & $648 \pm 14$ & $54.8 \pm 2.8$ \\
$\mathrm{HCO}_{3}(\mathrm{mmol} / \mathrm{L})$ & $26 \pm 0.6$ & $23.6 \pm 0.5$ \\
$\mathrm{Na}(\mathrm{mmol} / \mathrm{L})$ & $141 \pm 0.8$ & $141 \pm 0.6$ \\
$\mathrm{~K}(\mathrm{mmol} / \mathrm{L})$ & $3.1 \pm 0.0$ & $3.1 \pm 0.0$ \\
$\mathrm{Ca}(\mathrm{mmol} / \mathrm{L})$ & $1.5 \pm 0.0$ & $1.5 \pm 0.0$ \\
$\mathrm{Cl}(\mathrm{mmol} / \mathrm{L})$ & $130 \pm 0.8$ & $130 \pm 0.4$ \\
$\mathrm{O}_{2}$ content $(\mathrm{mL} / \mathrm{dL})$ & $1.94 \pm 0.04$ & $0.16 \pm 0.01$ \\
\hline$a C S F$, Artificial cerebrospinal fluid; $O G D$, oxygen-glucose deprivation.
\end{tabular}

$a C S F$, Artificial cerebrospinal fluid; $O G D$, oxygen-glucose deprivation. 